\title{
EL GÉNERO EN LA EDUCACIÓN PARA EL DESARROLLO SUSTENTABLE: REFLEXIONES DESDE LAS PRÁCTICAS PEDAGÓGICAS
}

\author{
SONIA SERRANO AROCHA \\ Antropóloga de la Universidad Nacional de Colombia. \\ Coautora del libro "Concepciones, prácticas y sentires pedagógicos de profesores universitarios: El caso de la Universidad Santo Tomás" . \\ Docente hora cátedra de las asignaturas Antropología en la cultura y Humanismo, cultura y valores. \\ Departamento de Formación Humanística. Universidad Pontifcia Bolivariana. \\ Bucaramanga. \\ ssserranoa@mail.com
}

\section{Cómo citar este artículo:}

Serrano, S. (2011). El género en la educación para el desarrollo sustentable: Refexiones desde las prácticas pedagógicas. Espiral, Revista de docencia e investigación. 1(1) 95 - 100

\begin{abstract}
Resumen
El presente artículo busca dar cuenta de la contribución de los análisis de género a la Educación para el Desarrollo Sustentable (EDS). Para tal fn se abordarán de manera refexiva los resultados de la investigación "Concepciones y prácticas pedagógicas de profesores universitarios: el caso de la Universidad Santo Tomás - Bucaramanga", fnalizada en el año 2010 donde participé como auxiliar de investigación. Específcamente haré referencia al apartado "El género en las prácticas pedagógicas universitarias", el cual constituye mi aporte al mencionado estudio.
\end{abstract}

Palabras clave: género, desarrollo sustentable, prácticas pedagógicas.

\begin{abstract}
This article seeks to review the contribution of gender analysis to Education for Sustainable Development. To reach this goal, we will have a thoughtful review of the results of the investigation "Pedagogical Conceptions and practices of university professors: the case of the Universidad Santo Tomás - Bucaramanga", fnished in 2010 where I participated as a research assistant. I refer specifcally to "Gender in university teaching practices," which is my contribution to this study.
\end{abstract}

Key words: genre studies, sustainable development, teaching practice.

\section{Introducción}

Se entiende el género según Karen Warren (2009) como

"la construcción social, por ejemplo, de la identidad y los roles de los seres humanos, basada en las concepciones del sexo biológico. Por lo general, el concepto género se utiliza, aunque no sin controversia, para distinguir el sexo biológico (masculino, femenino) de los conceptos de construcción social de los hombres y la masculinidad y de las mujeres y la feminidad"

En este sentido, esta categoría puede ser útil en el análisis de las prácticas pedagógicas ya que según Joan Scott en (Viveros, $2000,59)$ es un elemento constitutivo de las relaciones socioculturales basadas en las diferencias sexuales e inevitablemente ligado al ámbito simbólico, normativo, institucional y subjetivo de las personas.

Por esta razón, se encontró pertinente el aporte desde un análisis de género a la comprensión de las acciones educativas de los y las docentes, habida cuenta que los signif- 
cados, las interpretaciones y las expectativas del ser hombre o el ser mujer atraviesan diversos escenarios, entre los que se encuentran las instituciones educativas, así como las prácticas de los sujetos que pertenecen a ellas.

El género como categoría de análisis en la investigación "Concepciones y prácticas pedagógicas de profesores universitarios: el caso de la Universidad Santo Tomás - Bucaramanga" surgió de la refexión permanente del proceso metodológico junto con las investigadoras Sandra Patricia Basto y Brenda Isabel López, ya que en las observaciones directas en aula fueron evidentes ciertas particularidades en las actuaciones de los profesores y las profesoras. De acuerdo con Basto y López, el punto de partida para incorporar el género como categoría de análisis fue la presencia del modelo pedagógico conductista que, entre su variante tradicional y constructivista, fue evidente en las profesoras observadas. Una vez se encontró que las profesoras se ubicaban en la misma tendencia pedagógica, se dirigió la mirada hacia los profesores, quienes presentaron una tendencia general a trascender el modelo pedagógico tradicional o los esquemas conductistas, ubicándose en un modelo más alternativo. De esta manera, el estudio evidenció particularidades en las prácticas pedagógicas relacionadas con los roles de género en el ethos educativo de los profesores y profesoras.

Para comprender el anterior hallazgo es necesario acudir los Estudios de Género (EG), ya que a través de ellos, según Joan Scott (González, 2009:682), "se ha logrado incorporar el concepto de género, al análisis de las relaciones de poder existentes en un tiempo y lugar determinados". En este sentido, se hará mención de algunos aportes de los EG a la refexión de los procesos educativos.
En primer lugar, el papel que han cumplido los roles de género en el ethos educativo, es decir, la asignación social-cultural de funciones distintas a hombres y mujeres en las sociedades humanas, donde en la mayoría de los casos, los hombres realizan actividades "productivas" y las mujeres actividades relacionadas con lo "doméstico", se ha manifestado de manera similar en las actuaciones y prácticas de los y las docentes. Entre algunas autoras que sustentan lo anterior se encuentra Madeleine Arnot, $(1995,316)$ quien al estudiar diferencias de género en contexto educativo, a partir de la teoría política feminista, ha encontrado que existe una estructura histórica de la educación femenina basada en la diferencia: "mujeres y hombres somos distintos debido a la posición de las mujeres en el ámbito privado y público". Por otra parte, Imelda Arana (2001), a partir de un estudio sobre prácticas pedagógicas en maestros y maestras de un colegio del Distrito en Bogotá, encontró que se puede comprender la vida escolar local a partir del modelo propio de la división sexual y social del trabajo, así como que existen manifestaciones diferenciales en la práctica pedagógica de maestros y maestras en razón de las identidades de su sexo/género. Dos conclusiones relevantes del estudio de Arana son que: el magisterio es y será pensado, diseñado y ejecutado como práctica femenina, y que el modelo dominante en los procesos educativos y en la organización escolar es jerárquico y patriarcal, lo cual tiene efectos políticos considerables en términos de sobrepresentación de las maestras en situaciones de decisión y mando.

El otro aporte, considerado una crítica de los EG a la estructura de la educación, se manifesta a través de Ana Sacristán (1995,301), cuando explica aquel hecho conocido en la 
teoría feminista como "lo masculino como norma", referente a

“todo ese silenciamiento, exclusión de las mujeres, a la forma en que el conocimiento se ha construido desde puntos de vista, intereses y valores masculinos, a aquello que los hombres han sido y han hecho en distintos ámbitos a lo largo de la historia, a todo eso se ha considerado norma de validez universal y que en la educación este hecho no es la excepción, pues importantes obras de la historia tomaron la enseñanza de los hombres como modelo de referencia".

Es así como se problematiza la forma en que se ha educado en las sociedades occidentales contemporáneas: bajo el infujo de ideas androcéntricas, lo que ha implicado reproducir histórica, social y culturalmente desigualdades evidenciadas en valores, creencias, actitudes generalmente hacia las mujeres y traducidas en relaciones injustas de poder en todos los contextos.

\section{Discusión}

\section{La Educación para el Desarrollo Sustenta-} ble es un asunto de conciencia donde son relevantes los procesos educativos

Es necesario aclarar que en este artículo se usará la palabra sustentable y no sostenible. De acuerdo con Isabel Carrillo $(2006,130)$

"el vocablo inglés sustainable se utilizó en 1987 en el informe elaborado por la Comisión de Medio Ambiente y Desarrollo de las Naciones Unidas (WCED) para califcar el futuro al que se debería orientar la política social y económica mundial en relación con el medio ambiente".

Señala la autora que en la literatura en castellano a veces se encuentra como "sustentable" y otras como "sostenible", sin em- bargo, la diferencia es de uso regional ya que el término sustentable se utiliza generalmente en los países de habla hispana en América Latina, mientras que la palabra sostenible se emplea en España.

El Desarrollo Sustentable (DS) se entiende a partir de la WCED como aquel desarrollo que "satisface las necesidades del presente sin comprometer la capacidad de las generaciones futuras para satisfacer sus propias necesidades" (Warren, 2009, p. 104). Por tanto, la Educación para el Desarrollo Sustentable (EDS) requiere "integrar la temática (DS) a una agenda de aprendizaje, con el fn de asegurar un futuro sostenible". La EDS, fundamenta su existencia según Warren a partir de la Cumbre de la Tierra, celebrada en Río de Janeiro en 1992, en el marco de la Conferencia de la ONU sobre Medio Ambiente y Desarrollo (UNCED). En esta Cumbre se señala que:

a. "La educación es fundamental para que las personas promuevan el Desarrollo Sustentable y hagan frente a asuntos relacionados con Medio Ambiente y Desarrollo".

b. "La educación básica sirve de fundamento para la educación ambiental y el desarrollo, por tanto estos temas deben ser incorporados como parte esencial en el aprendizaje de las personas".

c. "La educación tanto formal como informal es indispensable para cambiar

las actitudes de las personas, para que tengan la capacidad de evaluar y abordar los problemas referentes al desarrollo sustentable con el $\mathrm{fn}$ de lograr una conciencia ética, valores, actitudes, habilidades y comportamientos coherentes con el desarrollo sustentable, que lleven a las personas a participar efectivamente en la toma de decisiones". 
De acuerdo a lo tratado en la WCED y en la Cumbre de Río, surgen varios temas para refexionar en el marco de los procesos educativos y, por tanto, de las prácticas pedagógicas. En primer lugar la EDS se puede considerar potencialmente una vivencia cultural, ya que se parte del supuesto de que todos los seres humanos se ven inmersos en procesos de aprendizaje gracias a las generaciones anteriores $y$ a instituciones más formales como la Escuela o la Universidad. Aun así, la garantía de un futuro sustentable en dicho proceso depende en gran medida del nivel de interiorización y de conciencia presente en las personas encargadas de los procesos de enseñanza.

Por otra parte delega y responsabiliza directamente a las instituciones, tanto de educación básica como de educación formal, entre las que se encuentran las Universidades, la labor de enseñar el medio ambiente. La refexión de esta tarea, más conocida como pedagogía del medio ambiente, implica según Pierre Giolitto desarrollar en los jóvenes un "sexto sentido" en relación con el medio ambiente y promover una verdadera instrucción cívica que permita plantear claramente los problemas ecológicos a la conciencia de los futuros ciudadanos, admitir algunos valores y clarifcar algunos conceptos con el fn de suscitar actitudes y aptitudes indispensables para la comprensión y apreciar las relaciones recíprocas que pueden existir entre el hombre, su cultura y su medio biofísico (Giolitto, 1984, p.135).

Ahora bien, si de conciencia se trata, la Carta de Belgrado (1975) defnió el primero de seis objetivos de la Educación Ambiental:

\footnotetext{
“conseguir que los individuos y los gru-
}

pos constituidos adquieran conciencia del medio ambiente global y de los problemas con él relacionados, y mostrarse sensibles respecto a él".

Como los asuntos de conciencia son construcciones sociales donde justamente la educación juega un papel muy importante, los demás objetivos tienen que ver con los conocimientos, valores y comportamientos que se deben construir con respecto a la temática del Medio Ambiente, entre los que se encuentran la responsabilidad crítica que genera participación inmediata en acciones para enfrentar las problemáticas medioambientales, y capacidades de evaluación y diseño de programas de formación relativos al medio ambiente en función de factores de orden ecológico, político, económico, social, estético y educativo.

\section{Aportes al análisis de las diferencias de género en las prácticas pedagógicas relativas a la Educación para el Desarro- llo Sustentable}

Las diferencias de género en las prácticas pedagógicas se referen a las formas diferenciadas en que profesores y profesoras proceden a compartir, transmitir y generar experiencias y refexiones de conocimiento en sus estudiantes. De acuerdo a lo explorado en la investigación "Concepciones y prácticas pedagógicas de profesores universitarios: el caso de la Universidad Santo Tomás - Bucaramanga", fueron evidentes algunos valores relacionados con los roles de género tradicionales asignados respectivamente a hombres y a mujeres, en el proceso de enseñanza de los profesores y las profesoras en el aula de clase. Este hallazgo constituye un punto de partida para pensar el papel que 
cumplen profesores y profesoras en las prácticas pedagógicas relativas a la Educación para el Desarrollo Sustentable, pues cabe mencionar que tradicionalmente la función de educar ha sido asignada a las mujeres y ligada a esta el valor de la responsabilidad, la atención y el cuidado de los/as otros/as.

Tal existir de las mujeres en función de los y las demás, ha terminado por incluir al medio ambiente. El Coloquio

de Aix-en-Provence (1972) ha sido una de las propuestas de la Educación Ambiental donde expertos defnieron acciones para adquirir actitudes y aptitudes para dar a conocer y respetar el medio ambiente, basadas en valores en su mayoría intrínsecamente femeninos. Se insistía en la idea de que "cada alumno entrara en contacto con los medios más diversos, a fn de llevarlo sucesiva y simultáneamente a mirar, acoger, observar, conocer, comprender, amar, proteger, protegerse y transformar" (Giolitto, 1984, p. 142).

En Aix se establecieron varias ideas sobre la pedagogía del medio ambiente, que pueden ser debatidas desde el análisis de género. La propuesta consistía fundamentalmente en

“generar en los estudiantes actitudes de responsabilidad, sensibilidad y conciencia frente a la naturaleza y la vida, a partir del conocimiento de los mecanismos que rigen el funcionamiento del medio ambiente biológico, histórico, socioeconómico y cultural" (Giolitto, 1984, p. 147),

pero difícilmente se pensó que dichos mecanismos de ese funcionamiento tienen arraigadas estructuras desiguales de poder que corrientes futuras del pensamiento feminista, como el ecofeminismo, podrían cuestionar esas intenciones.

Según Warren (2009), el ecofeminismo

"usa el análisis de género para abordar las diferentes formas de dominación injusta de las mujeres y otros humanos (personas negras, pobres, analfabetas, de diferentes etnias, y demás) como cuestiones que han estado históricamente interconectadas con la, también injusta, dominación de la Tierra, o el medio ambiente no humano. Estas interconexiones pueden ser históricas, empíricas, socioeconómicas, conceptuales, lingüísticas, simbólicas y literarias, éticas, teóricas, espirituales, religiosas, teológicas y epistemológicas".

Esta corriente surge y puede refejar diferentes perspectivas feministas de acuerdo a las diferentes interconexiones, así que no es de extrañar que exista más de un signifcado para el término. Más bien, señala Warren(2009):

“es un término genérico para un movimiento social y la teoría afrma que los problemas ambientales tienen una relación directa con, y mutuamente reforzada por, los sistemas injustifcados de dominación humana $\mathrm{y}$, para que sean seriamente abordados se deben tener en cuenta categorías como el género, la raza o las cuestiones étnicas, socioeconómicas, geográfcas y coloniales". (38)

Se aborda entonces el ecofeminismo como una herramienta útil para cuestionar las problemáticas medioambientales en términos de justicia social, así como en términos explícitamente incluyentes de otros seres vivos no humanos y la naturaleza en el análisis de los asuntos de las mujeres. 
En el contexto del Desarrollo Sustentable y la Educación para el Desarrollo Sustentable con perspectiva de género, el ecofeminismo se centra en las mujeres de diferentes razas o etnias, clases sociales, ubicación geográfca, y lugares históricos con el fn de revelar características de la dominación de género que infuyen en las cuestiones relativas al medio ambiente y al desarrollo, lo que genera graves consecuencias en relación al acceso de las mujeres al agua, a la propiedad de la tierra, al desarrollo traducido en proyectos productivos y acceso a información y conocimiento donde el principal medio es la educación.

Algunas refexiones sobre las prácticas pedagógicas de profesores y profesoras en cuanto a la Educación para el Desarrollo Sustentable tienen que ver con:

Readaptar las propuestas mundiales de la Educación Ambiental que tome en cuenta las interconexiones planteadas por Karen Warren: análisis de género, raza, cuestiones étnicas, socioeconómicas, geográfcas y coloniales.

Pensar la Educación Ambiental específcamente desde la Universidad al indagar la responsabilidad individual crítica, de la que habla Pierre Giolitto en el caso de la Educación Superior, como una etapa de la formación de los individuos y orientándola a un tipo de responsabilidad social ambiental que implique el papel activo de los y las docentes en la construcción colectiva de tareas, compromisos, proyectos de investigación e interAbstract

This article is a pedagogical exercise mediated by a

vención medio ambiental con los y las estudiantes, independientemente del género.

Transversalizar la temática del Desarro1lo Sustentable en el currículo, a partir del hecho que menciona Giolitto: "todos los aspectos de la vida humana (físicos, químicos, biológicos, sociológicos, económicos y culturales) repercuten en el Medio Ambiente".
Convertir la asignación única (histórica, social y cultural) a las mujeres del valor de la responsabilidad y el sostenimiento de la sociedad, que incluye al Medio Ambiente, en una asignación colectiva, de acuerdo con una lógica más igualitaria de responsabilidades tomada la educación como punto de partida para garantizar realmente la continuidad del proceso de transformación que plantea la Educación Ambiental a nivel mundial hace décadas.

\section{Referencias bibliográfcas}

Arana, I. (2001). Las prácticas pedagógicas de maestras y maestros del Distrito Capital. Una mirada a los roles de género. Nómadas, 14, 90- 101.

Arnot, M. (1995). Feminismo y educación democrática. En Volver a pensar la educación. Vol. I. Madrid: Morata.

Carrillo, I. (2006). Ecología Urbana y Desarrollo Sustentable de las ciudades. En Alderoqui, S. \& Pechansky, P. (Comp.). Ciudad y Ciudadanos. Aportes para la enseñanza del mundo urbano. Buenos Aires: Paidós.

Giolitto, P. (1984). Pedagogía del Medio Ambiente. Principios de ecología. Barcelona: Herder.

González, M. (2009). Estudios de Género en Educación. Revista Mexicana de Investigación Educativa, 14 (42), 681-696. Descargado julio 18, 2010 de http:// www. comie.org.mx/v1/revista/portal.php?idm $=$ es\&sec $=$ SC01\&sub $=$ SBA\&criterio $=$ N042

Sacristán, A. (1995). Apuntes críticos sobre feminismo y educación. En Volver a pensar la educación. Vol. I. Madrid: Morata.

Viveros, M. (2000). Notas en torno a la categoría de género. En Robledo, A. \& Puyana, Y. (Comp.). Ética: masculinidades y feminidades. Bogotá: Universidad Nacional de Colombia.

Warren, K. (2009). Sustainable Development and Sustainable Development Education: An Ecofeminist Philosophical Perspective on the Importance of 
